A Multi-Agents Platform for a Corporate Web

\title{
Semantic
}

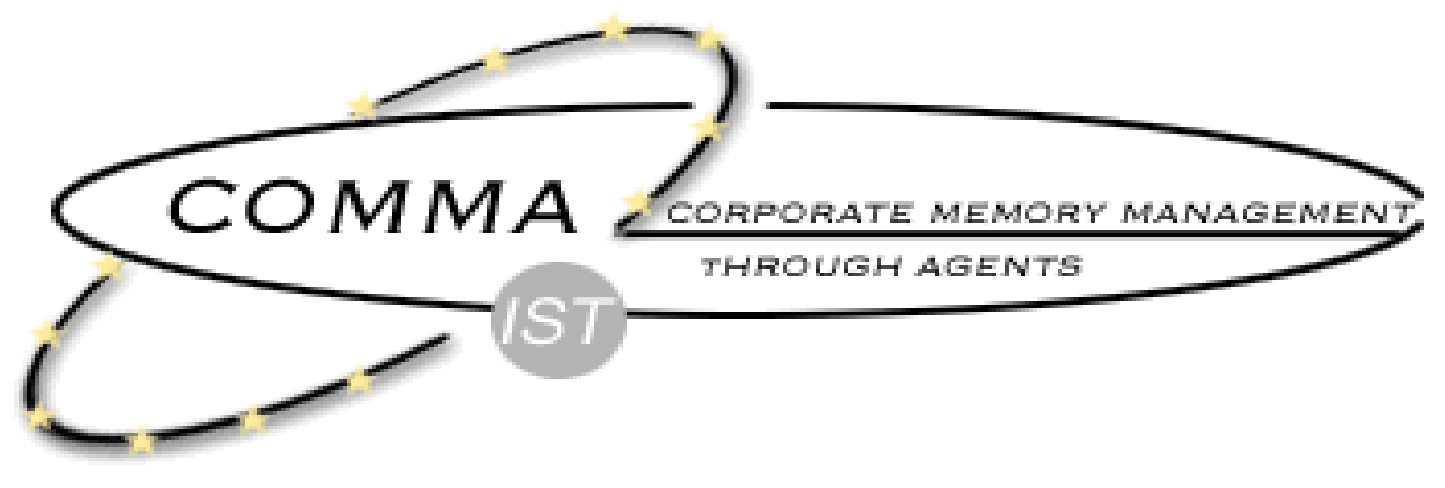




\section{A Multi-Agents Platform for a Corporate Web Semantic}

Corporate memory materialization in CoMMA

- Objectives \& Overall Approach

- Use of RDF(S) to build a corporate semantic web

- CoMMA MAS architecture overview

- Handling distribution of annotations

$\checkmark$ Allocating new annotation

- Distributed query-solving 
COMMA - Euronoon nroiont - Drovido what is CoMMA?

CoMMA: European project : Provide a corporate memory management framework. Started February 2000 - Ended February 2002 2 application \& trial scenarios

$\checkmark$ Assist new employee integration

- Support technology monitoring activities 
CoMMA: European project : Provide a

corporate memory management framework.

Started February 2000 - Ended February 2002

Positioning \& Approach

- Corporate memories as heterogeneous and distributed information landscapes

- Stakeholders are a heterogeneous and distributed population

- Exploitation of corporate memory involves heterogeneous and distributed tasks

\section{Materialization CM Exploitation}

XML: Web standard, Structure, Extend, Validation, Transform RDF(S) \& K. Eng.: Annotation, Schemas

\section{Multi-Agent System:}

Modularity, Distributed, Collaboration, Sem. M.P.

\section{Machine Learning:}

Adaptability

4 Fabien.Gandon@sophia.inria.fr $\quad$ A Multi-Agents Platform for a Corporate Web Semantic 


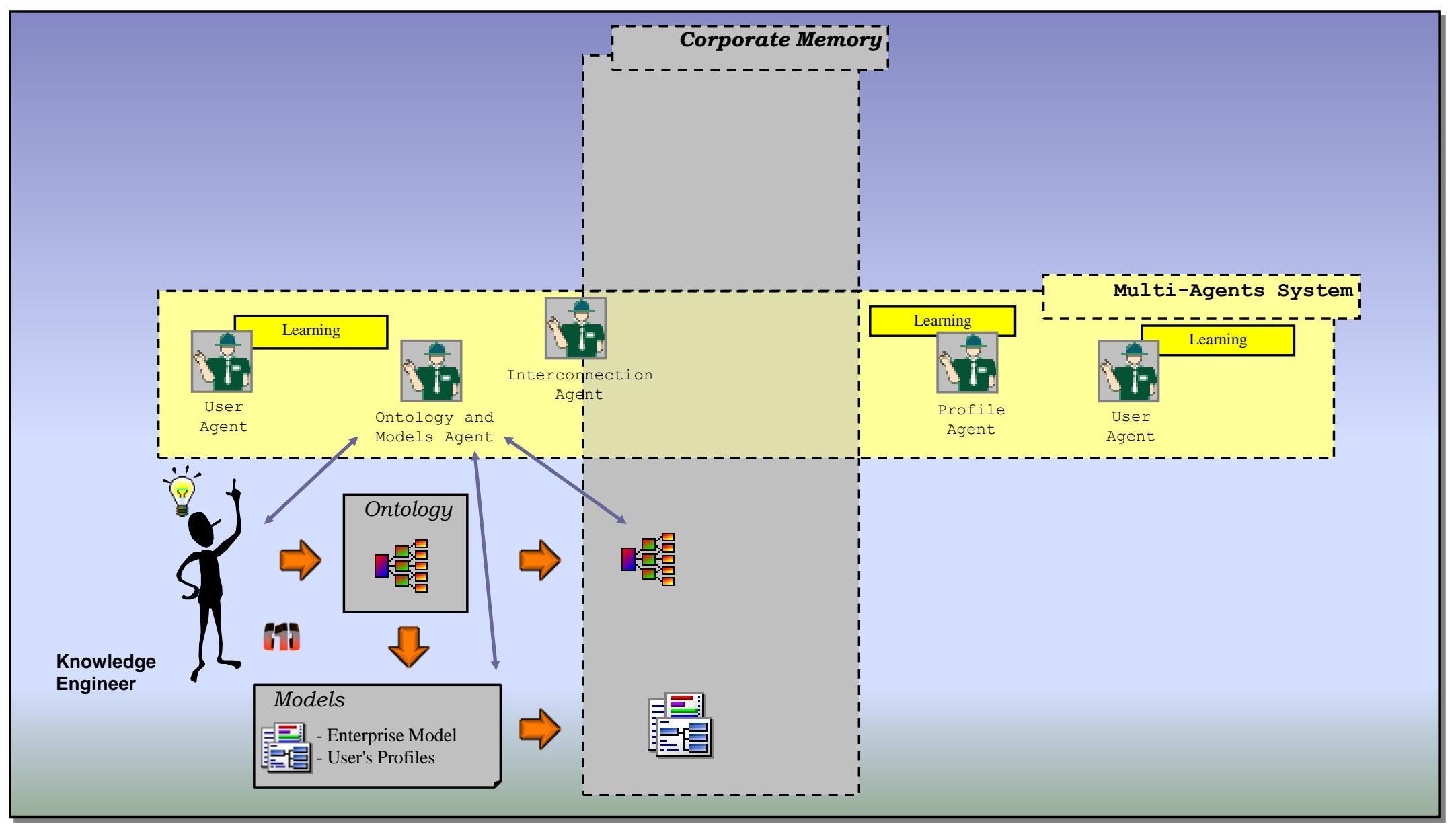




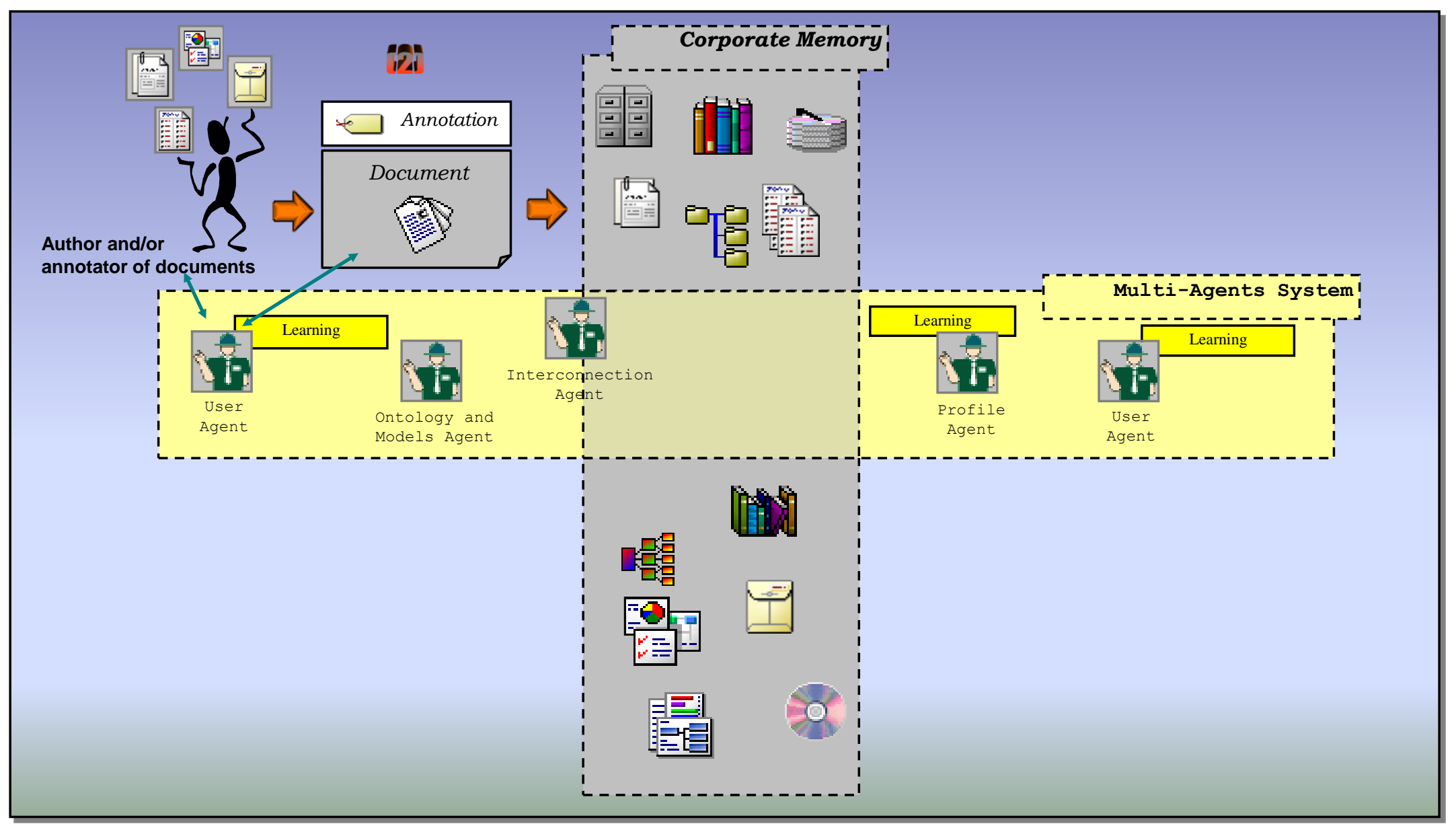




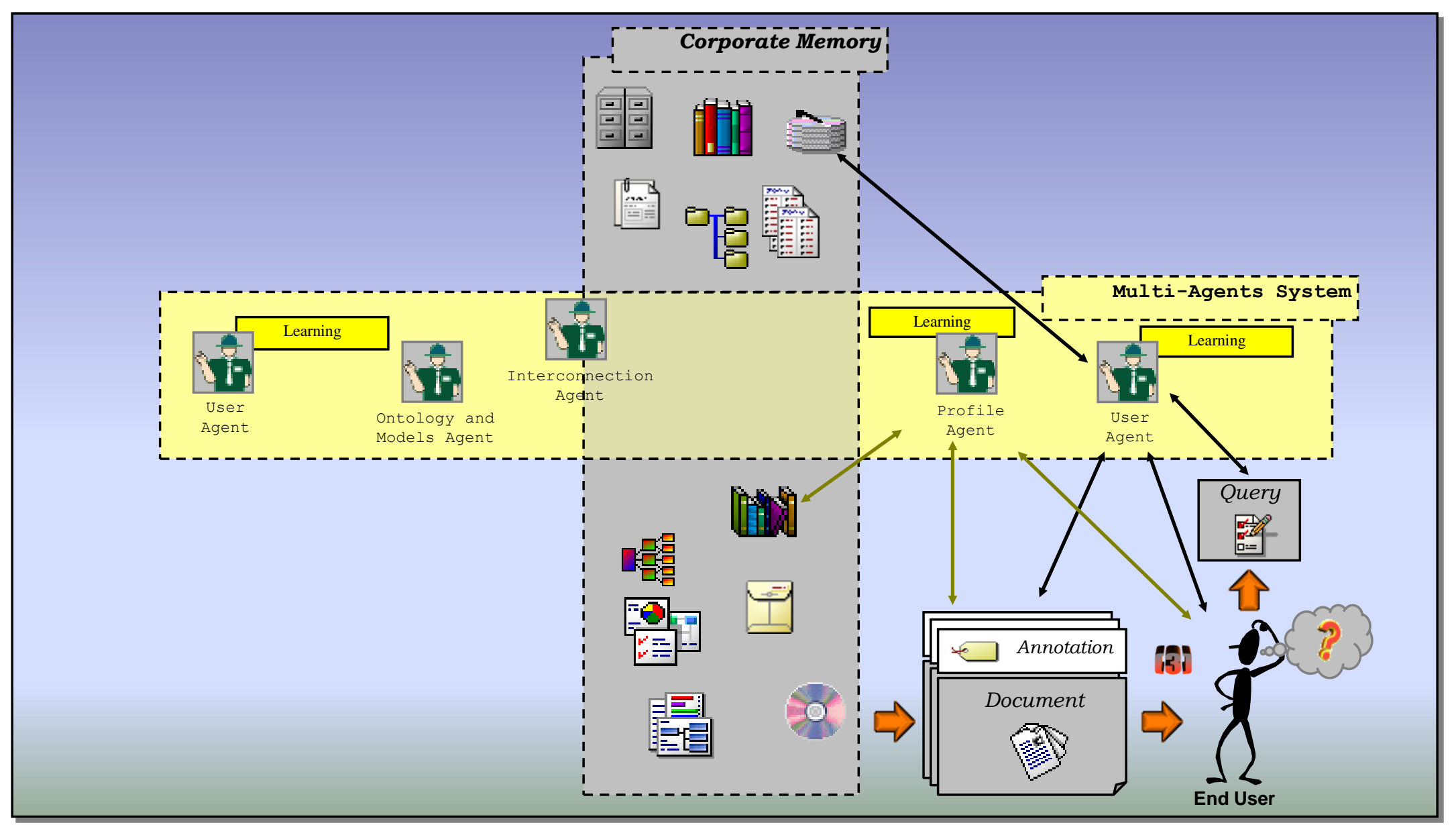




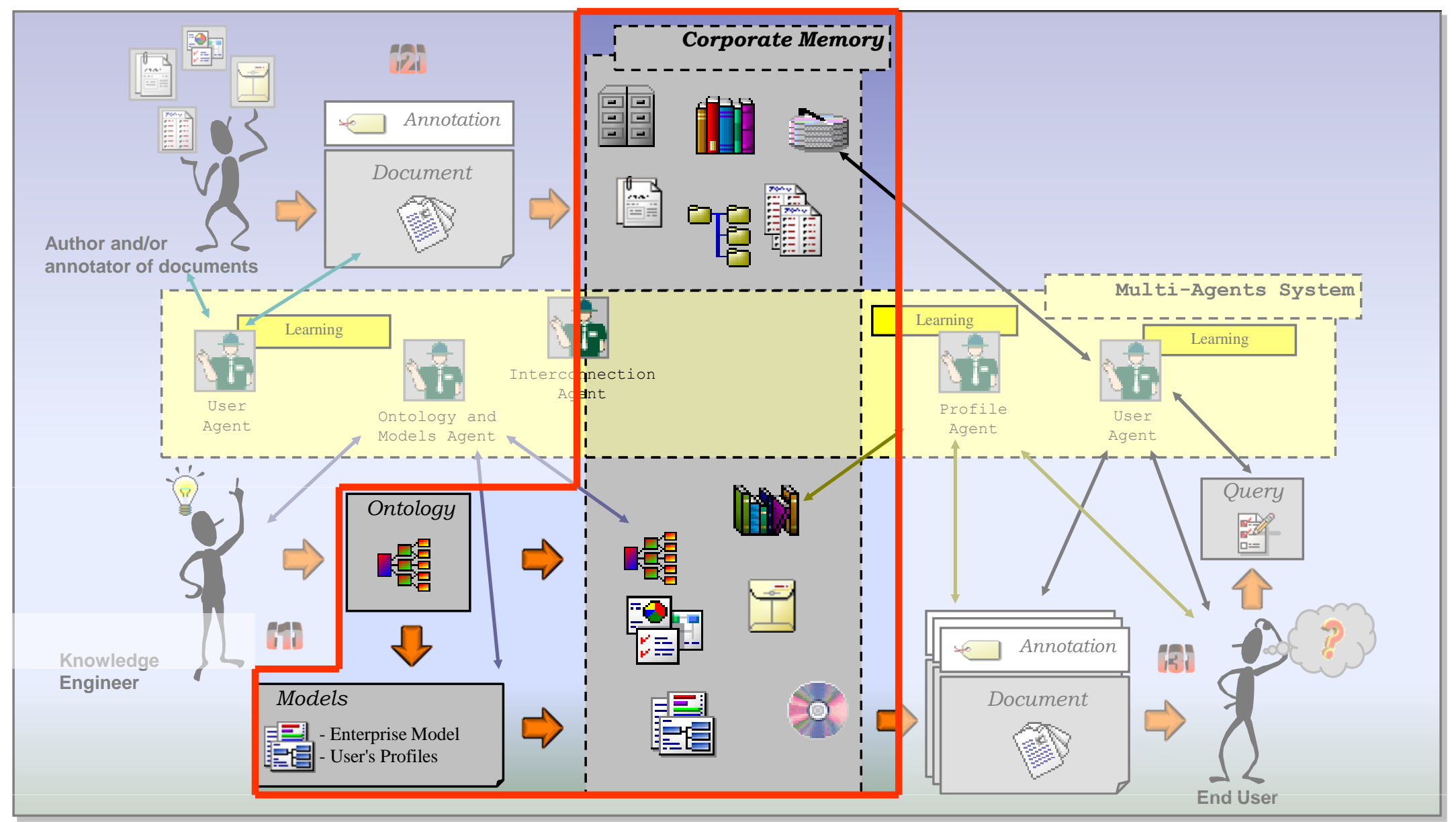

8 Fabien.Gandon@sophia.inria.fr

A Multi-Agents Platform for a Corporate Web Semantic 


\section{A Corporate Semantic WWb}

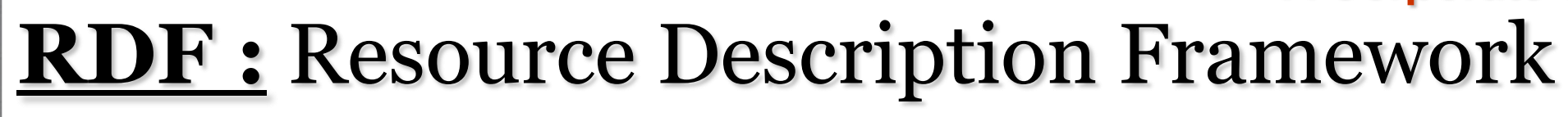

- Describe Web resources

- RDF Schema (to formalize the ontology)

\section{Approach :}

- ontology in RDFS (O'CoMMA)

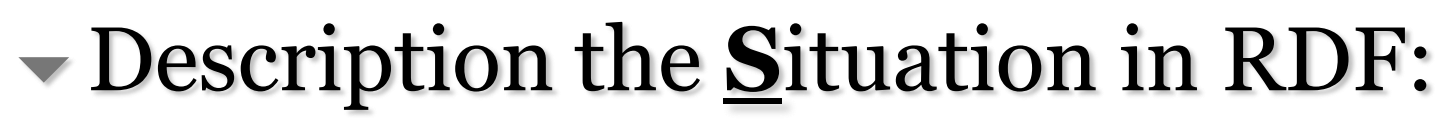

$\square$ User Profiles

$\square$ Organization model

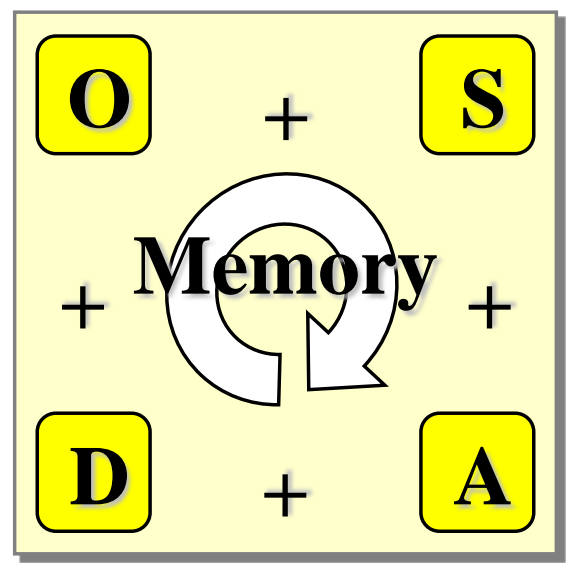

- Annotations in RDF describing Documents

- Toward a corporate semantic web

- Annotated world for agents (quickly intelligent) 


\section{RDF(S) - Aspects used by the agents managing the annotations}

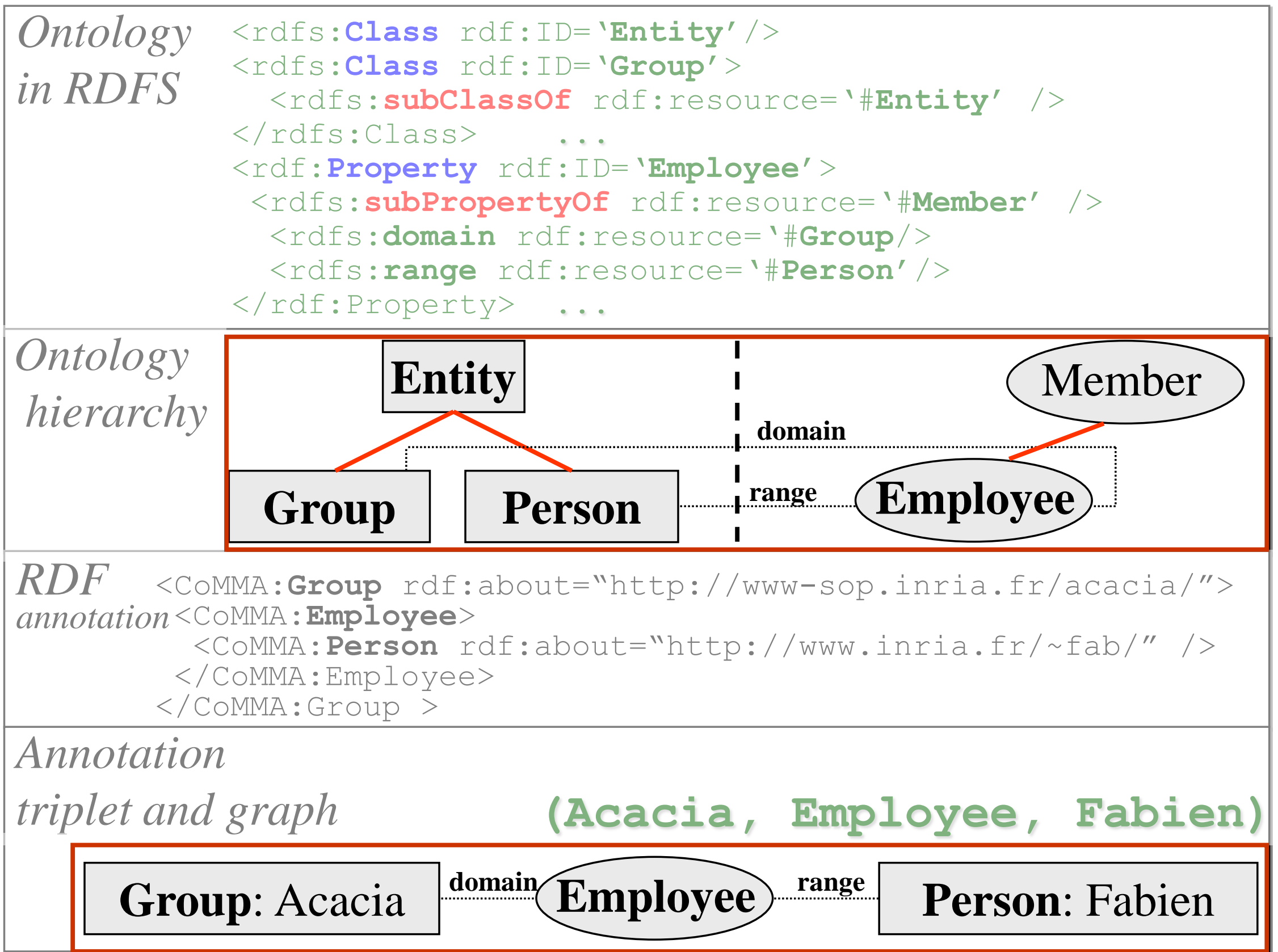




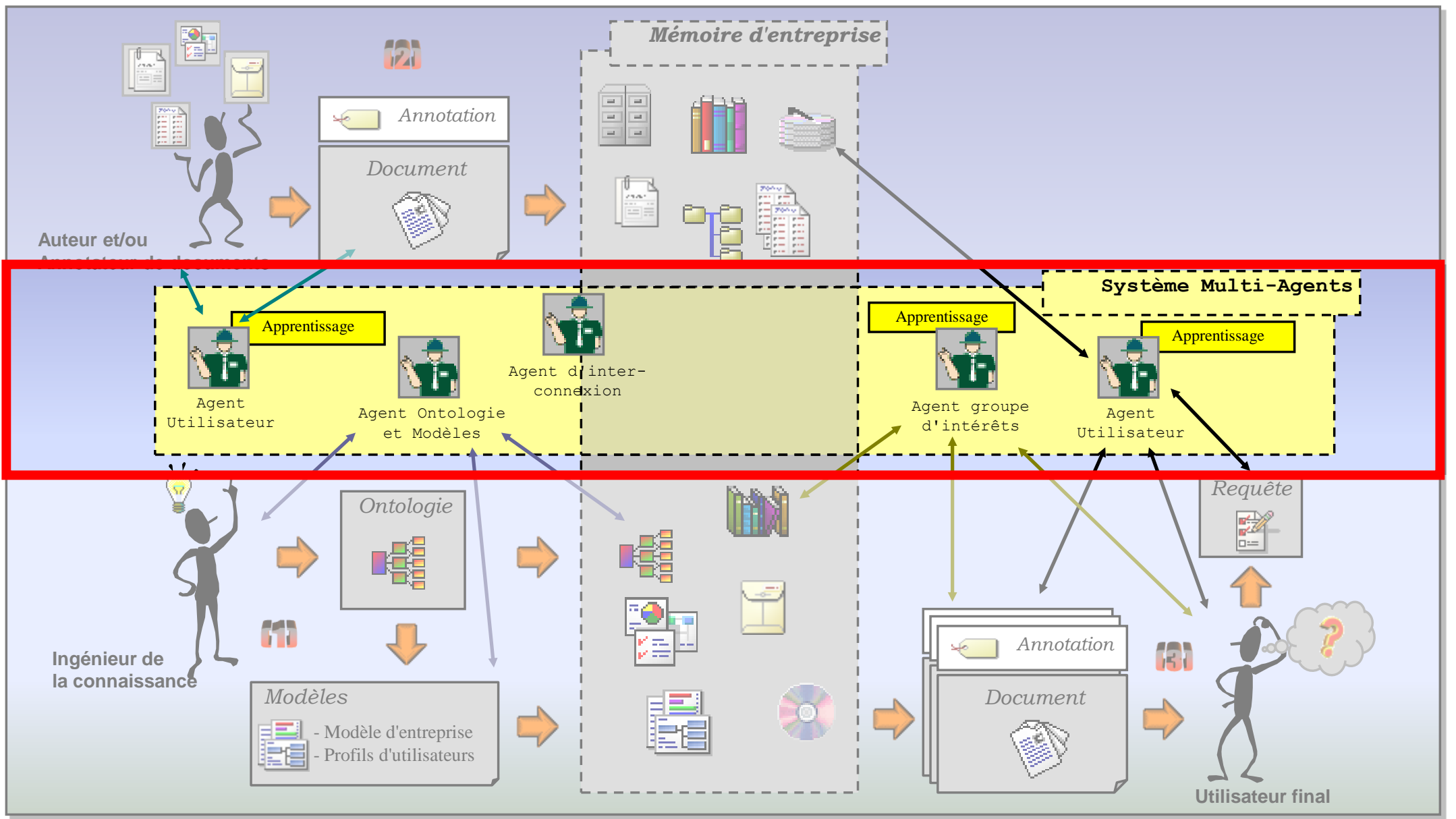


Multi-agents information system for the CM

CoMMA is an heterogeneous multi-agents information system

- Several types of agents

- Deal with duality of information distribution:

$\square$ i.e. scattered data, information \& knowledge

$\square$ i.e. diffuse captured information and knowledge

- Agent paradigm adequacy:

- Collaboration $\rightarrow$ Global Capitalization

$\square$ Autonomy \& Individuality $\rightarrow$ Local Adaptation

\section{From Macroscopic to Microscopic}

- Functional analysis for high level functions: societies

- Society internal functional analysis: roles

$\checkmark$ in // scenario and use-cases analysis: interactions 


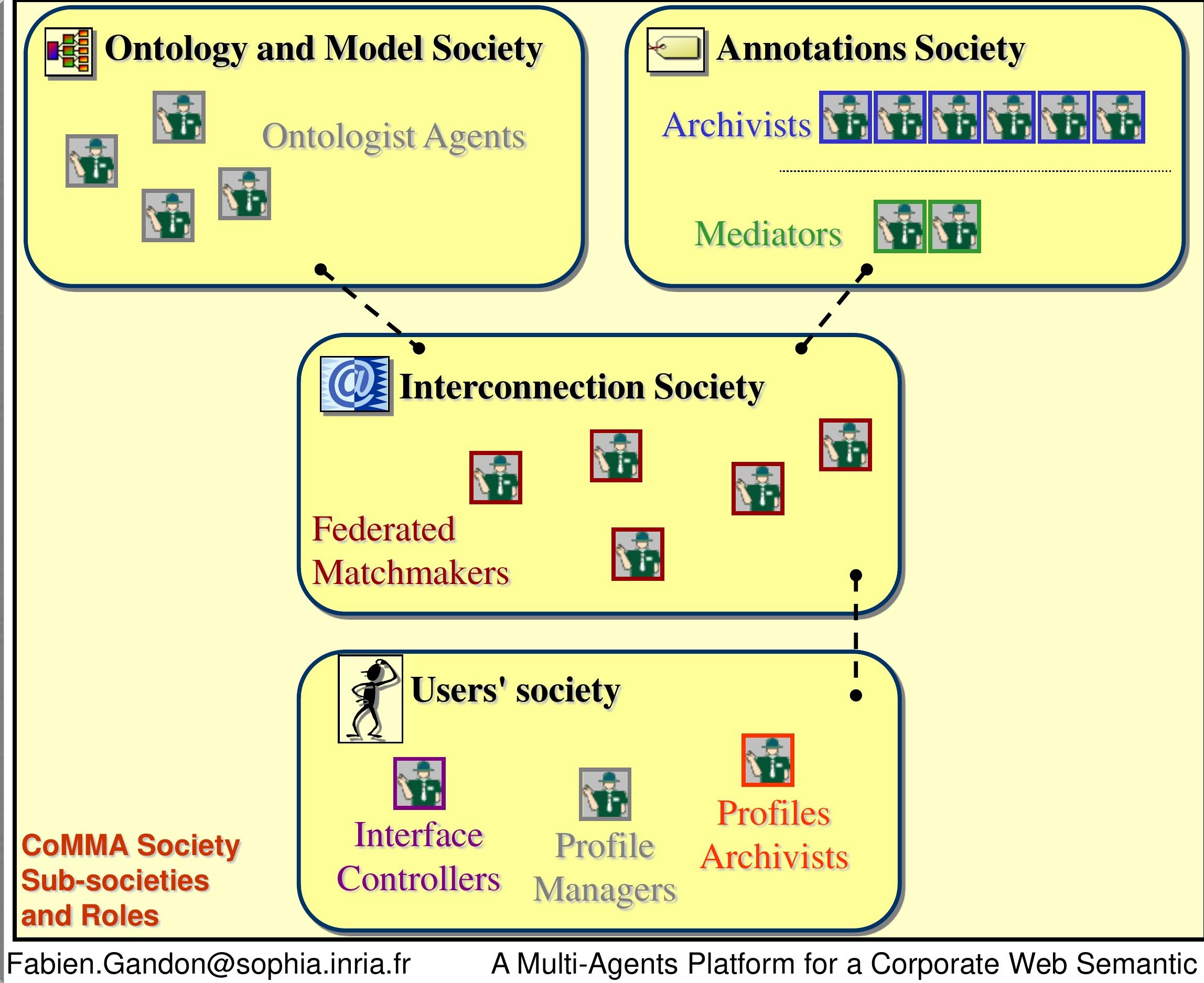




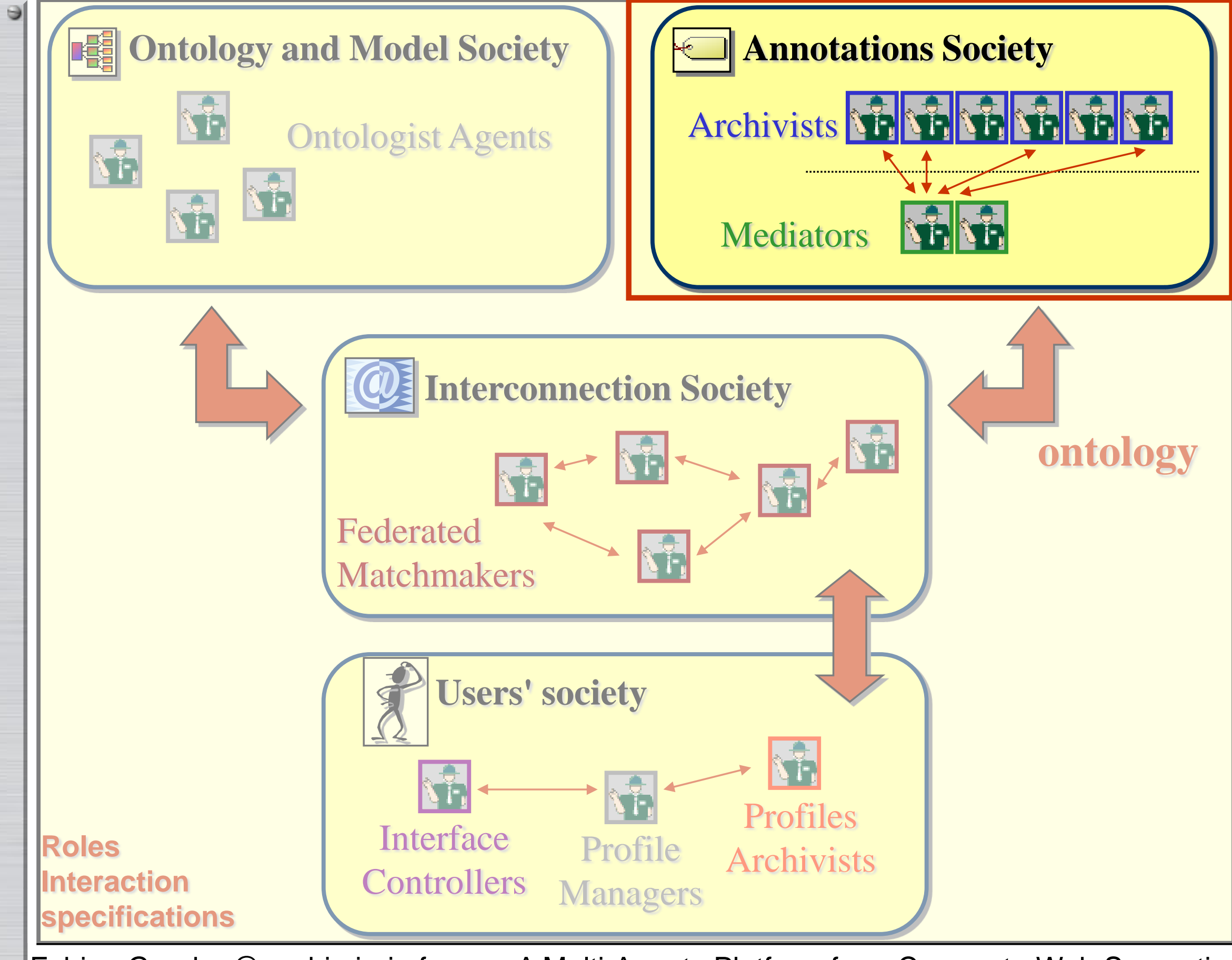


Zooming on the annotation society

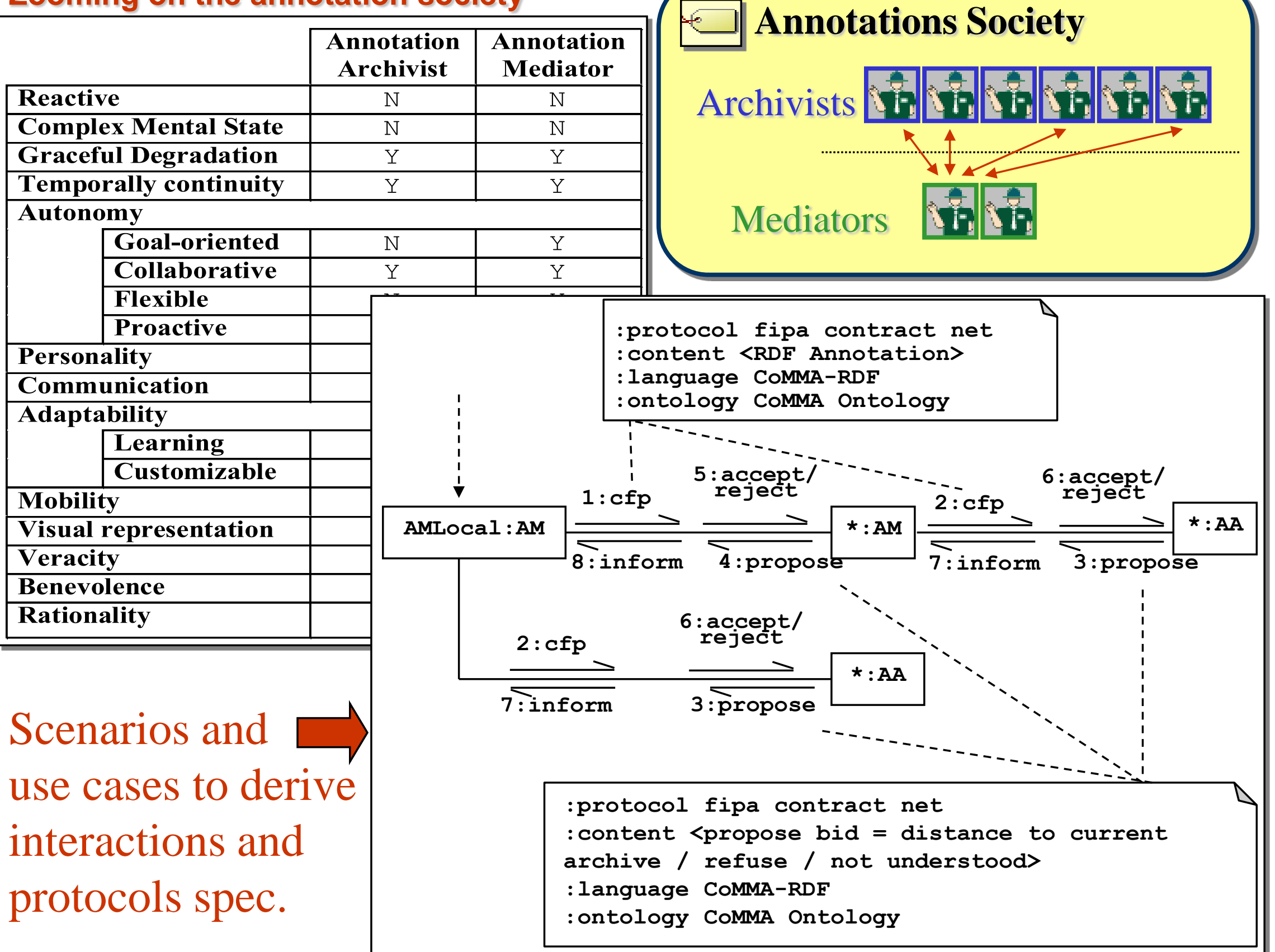


Interactions AA-AM I I allocating a nanotation C-Net : Annotation allocation

- AM - AA discuss best place to archive

- Contract-net (CfP, Proposal, Accept/Reject)

- Allocation criteria: pseudo-semantic measure

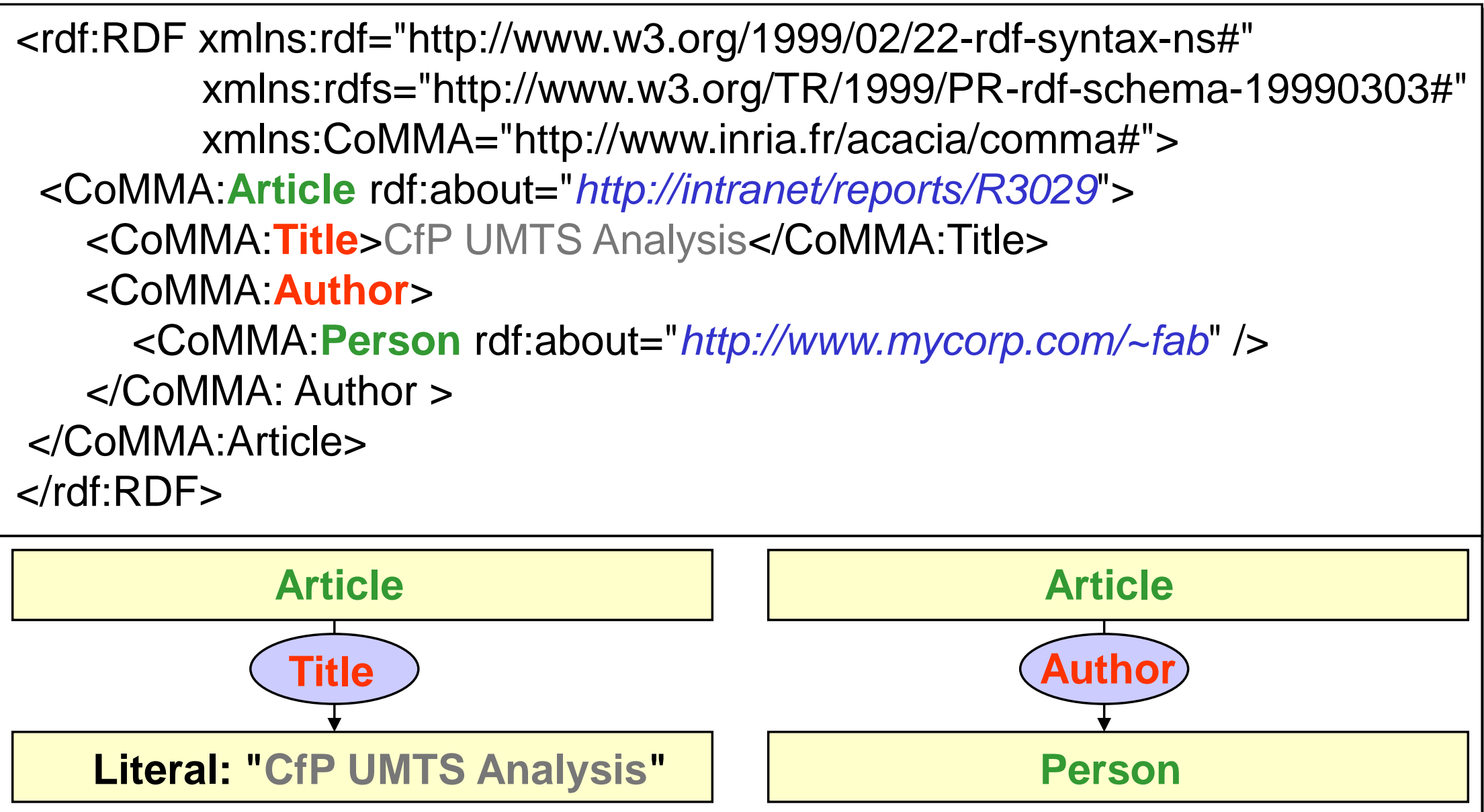


Interactions AA-AM in allocating an annotation

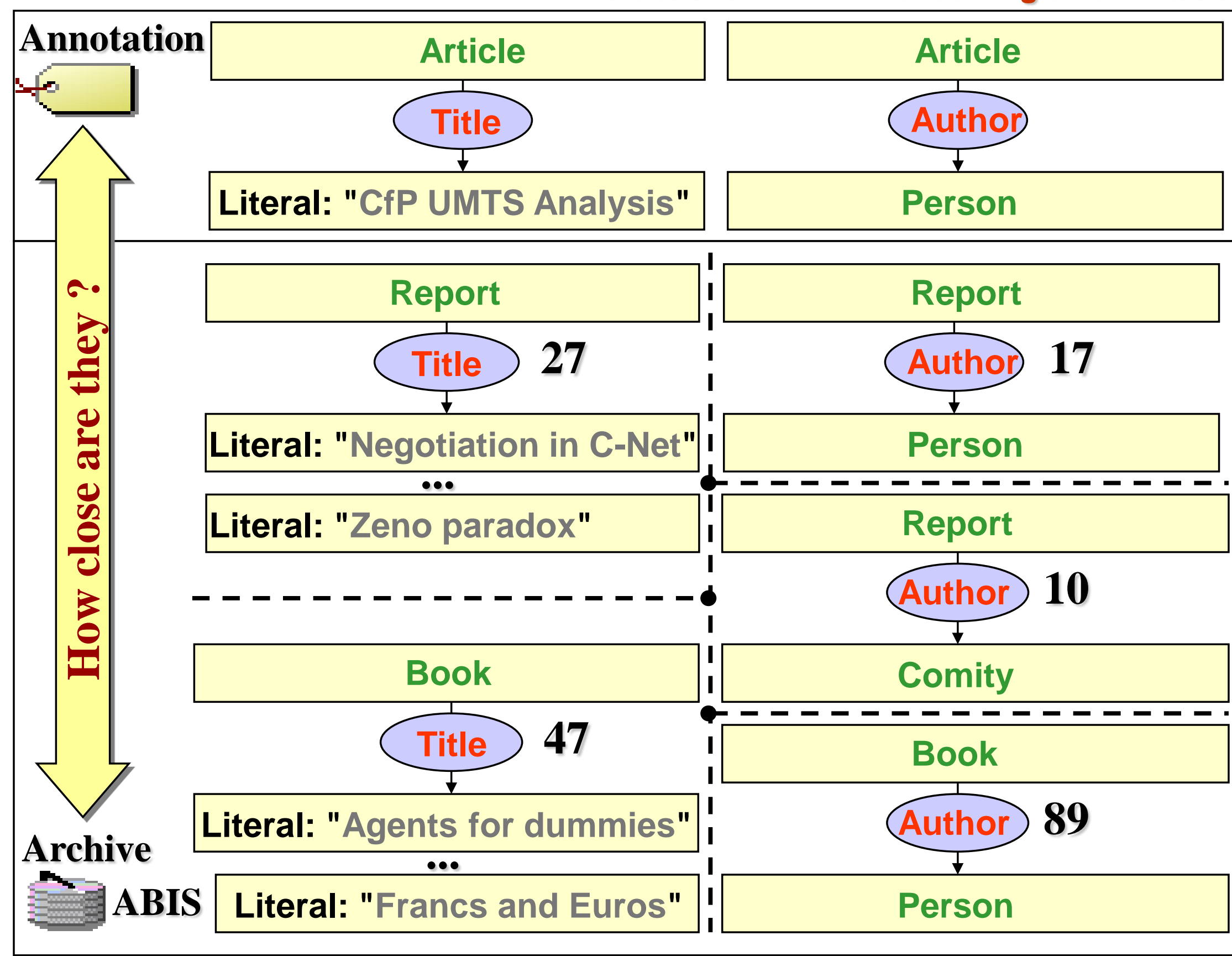


ㅂ Allocating an annotation: lexicographical distance
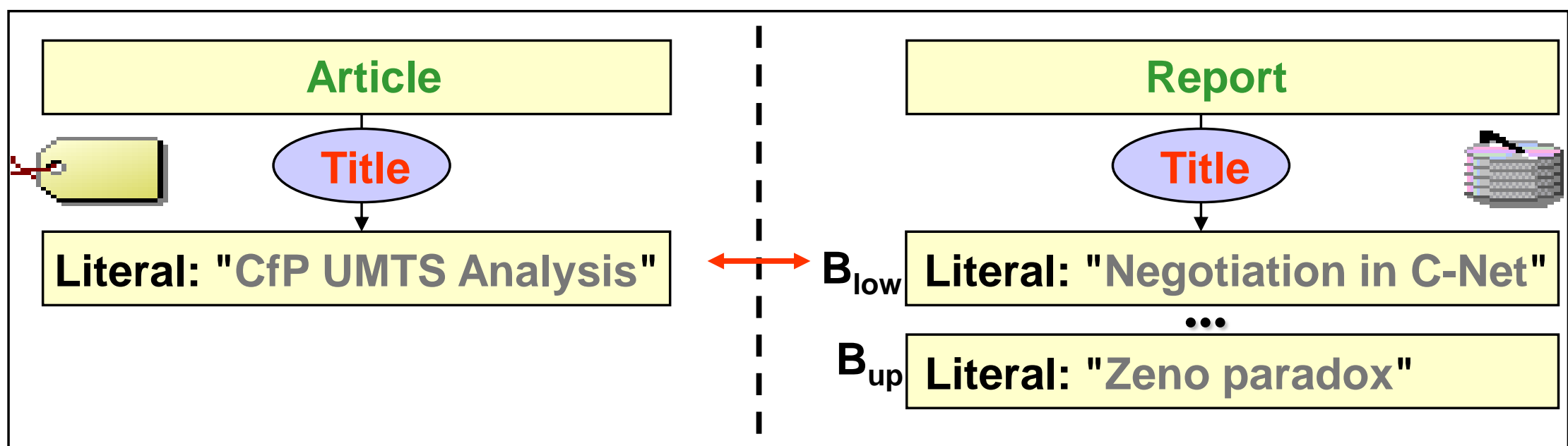

Classic lexicographical distance:

$(\Rightarrow$ pseudo semantic)

$\operatorname{Abscissa}\left(\operatorname{Lit}_{\mathrm{X}}\right)=\sum_{i=0 . s} \frac{C_{\mathrm{x}, \mathrm{i}} \operatorname{Max}_{\mathrm{L}}{ }^{i}}{{ }^{2}} \in\left[0, \operatorname{Max}_{\mathrm{L}}\left[; \operatorname{Lit}_{\mathrm{X}}=C_{\mathrm{x}, 1} \ldots C_{\mathrm{x}, \mathrm{s}}\right.\right.$

$\operatorname{Dist}_{\mathrm{L}}\left(\operatorname{Lit}_{\mathrm{A}}, \operatorname{Lit}_{\mathrm{B}}\right)=\left|\operatorname{Abscissa}\left(\operatorname{Lit}_{\mathrm{B}}\right)-\operatorname{Abscissa}\left(\mathrm{Lit}_{\mathrm{A}}\right)\right|$

Distance to a literal interval: $(\Rightarrow$ pseudo distance)

$$
\begin{aligned}
& \operatorname{Dist}_{\mathrm{I}}\left(\operatorname{Lit}_{\mathrm{X}},\left[\mathrm{B}_{\text {low }}, \mathrm{B}_{\text {up }}\right]\right) \\
& \quad \text { if } \mathrm{Lit}_{\mathrm{X}} \in\left[\mathrm{B}_{\text {low }}, \mathrm{B}_{\text {up }}\right] \text { then }=0 \\
& \quad \text { else }=\operatorname{Min}\left(\operatorname{Dist}_{\mathrm{L}}\left(\operatorname{Lit}_{\mathrm{X}}, \mathrm{B}_{\text {low }}\right), \operatorname{Dist}_{\mathrm{L}}\left(\mathrm{Lit}_{\mathrm{X}}, \mathrm{B}_{\text {up }}\right)\right)
\end{aligned}
$$



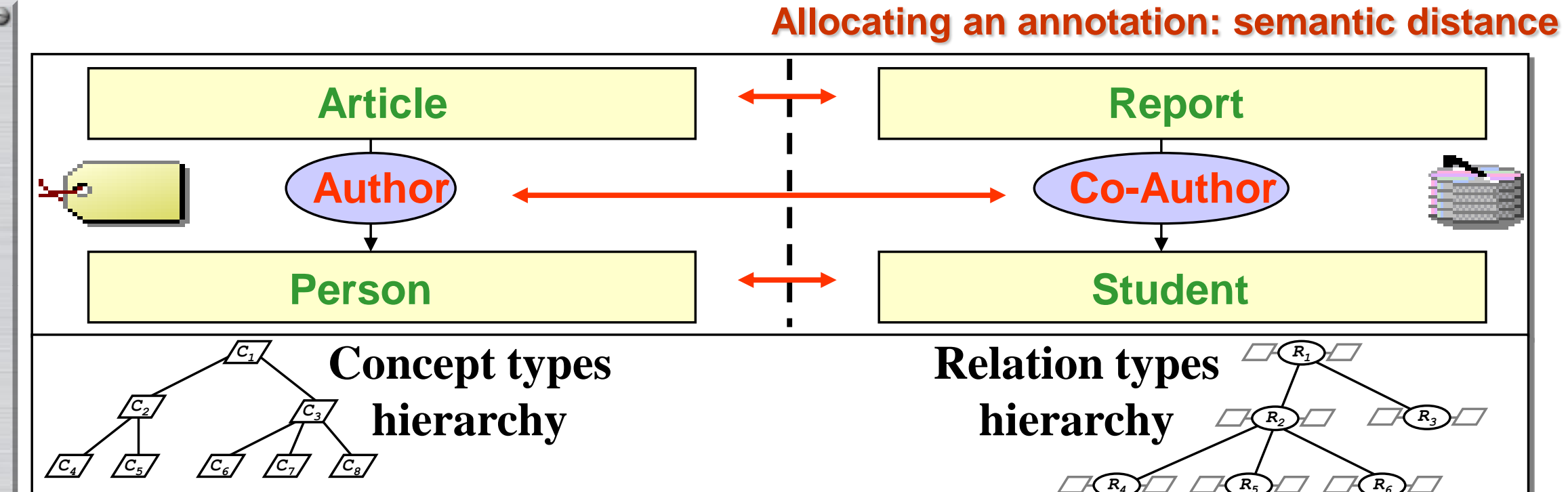

Distance from Type ${ }_{1}$ to Type ${ }_{2}$ through least common super-type

$\operatorname{Dist}_{\mathrm{H}}\left(\right.$ Type $\left._{1}, \mathrm{Type}_{2}\right)=\operatorname{SPath}\left(\right.$ Type $_{1}$, LCST $)+$ SPath $\left(\right.$ Type $_{2}$, LCST $)$

SPath(,): number of edges through generalisation links

LCST: least com

Dist $_{\mathrm{TL}}\left(\right.$ Type $\left._{1}, \mathrm{Lit}_{\mathrm{X}}\right)$

Triple-triple: condi

Dist $_{\text {TFABIS }}\left(\right.$ Triple $_{A}$,

Dist $_{\mathrm{Ci}}=$

$\mathrm{W}_{\mathrm{C}}{ }^{*} \mathrm{Di}$

Distance(coupé,wagon) $=2$

$$
\text { or } \mathrm{W}_{\mathrm{C}} * \mathrm{Di}
$$

or $\mathrm{W}_{\mathrm{L}} * \mathrm{~N}$

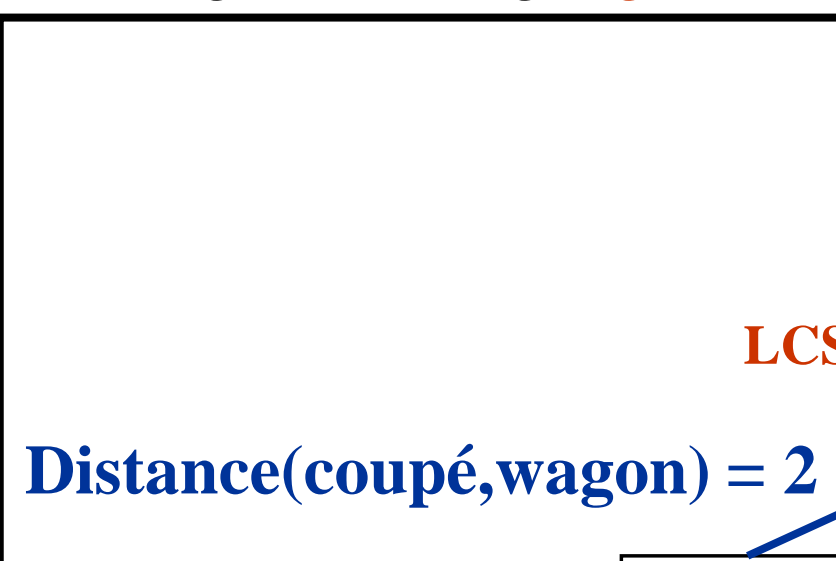

Toy Example vehicle

car

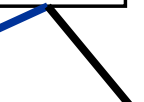

wagon

DIST $_{\text {ILIl, }}\left[\mathrm{D}_{\text {low }}, \mathrm{D}_{\text {up }}\right]$ )

Iv-IVIax ${ }_{C}$ z/IVIax bicycle

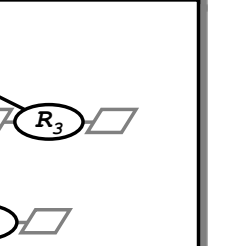

Relation types hierarchy 
Annotation $-\square \longrightarrow$ ABIS

Dist $_{\text {AABIS }}($ Triple,$A B I S)=\operatorname{Min}\left(\right.$ Dist $_{\text {TFABIS }}\left(\right.$ Triple, Triple $\left._{\mathrm{i}}\right)$

Triplet $_{\mathrm{i}} \in$ ABIS

Dist $_{\text {AABIS }}\left(\mathrm{An}_{\mathrm{X}}, \mathrm{ABIS}\right)=\sum$ Dist $_{\mathrm{TABIS}}\left(\right.$ Triple $\left._{\mathrm{j}}, \mathrm{ABIS}\right)$

Triple $_{j} \in A n_{X}$

Dist $_{\mathrm{ACAP}}\left(\mathrm{An}_{\mathrm{X}}, \mathrm{CAP}\right)=\sum$ Dist $_{\mathrm{TCAP}}\left(\right.$ Triple $\left._{\mathrm{j}}, \mathrm{CAP}\right)$

$$
\text { Triple }_{j} \in A n_{X} \quad \text { sub-type } \Rightarrow \text { Dist }=0
$$

$\operatorname{Dist}\left(A_{\mathbf{X}}, A_{A_{Y}}\right)=\operatorname{Dist}_{\mathrm{AABIS}}\left(\mathrm{An}_{\mathrm{X}}, \mathrm{ABIS}_{\mathrm{Y}}\right)+\operatorname{Dist}_{\mathrm{ACAP}_{\mathrm{P}}}\left(\operatorname{An}_{\mathrm{X}}, \mathrm{CAP}_{\mathrm{Y}}\right)$

\section{Allocation criteria:}

- Winner $=$ Archivist with the smallest distance

- Effect: cluster annotations having close semantic contribution $\rightarrow$ specialise the archives

- One use: specialisation improve query solving and respect knowledge distribution 


\section{Fragmentation and distributed queries}

- Co-operatively solve a query (multi-stage Query-Ref)

- AM decomposes submitted query into sub-queries

- Allocation of sub-query based on ABIS

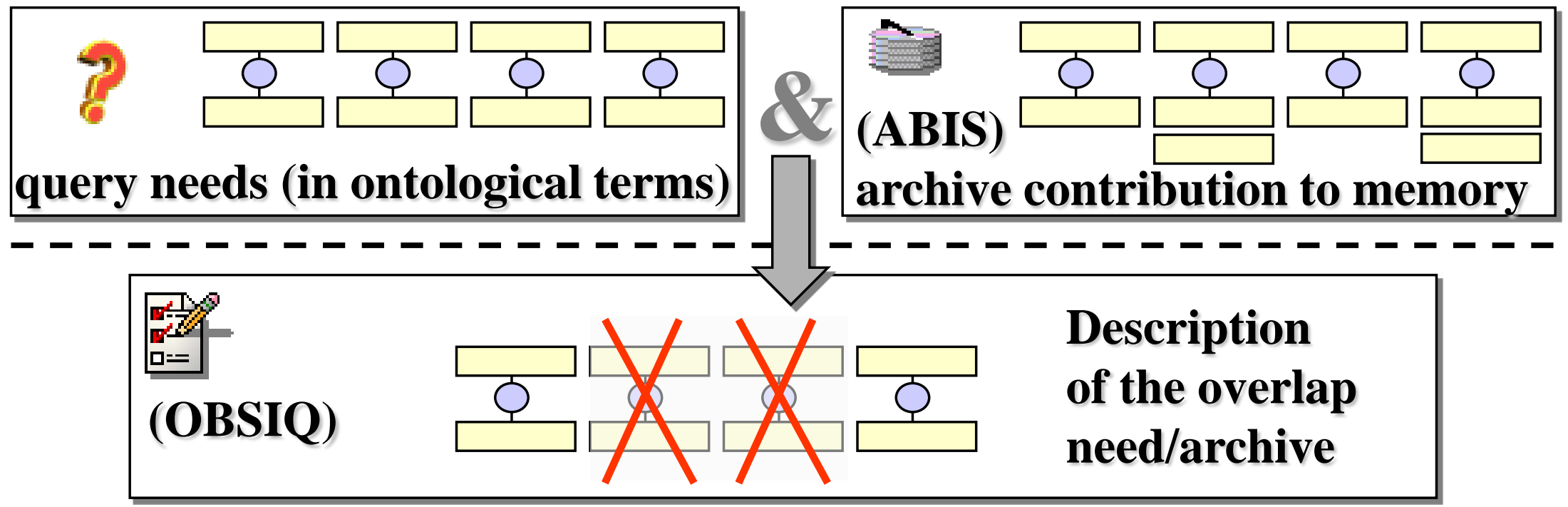

\section{Overlap description}

- Refines service description of Archivists

- Target multicast communications in query-solving

- Exploit archive specialisation obtained by the distribution of annotations 
$\theta \mid<$ xml version="1.0" encoding="UTF-8"?>

<rdf:RDF xmlns:rdf="http://www.w3.org/1999/02/22-rdf-syntax-ns\#"

DOM RDF structure xmlns:rdfs="http://www.w3.org/TR/1999/ PR-rdf-schema-19990303\#"

xmlns:CoMMA="http://www.inria.fr/acacia/comma\#">

$<\mathrm{COMMA}:$ Document $>$

$<$ COMMA : CreatedBy $>$

$<$ COMMA : Person $>$

$<$ CoMMA: FamilyName>?AuthorName</CoMMA: FamilyName>

$<$ CoMMA: FirstName $>$ ? AuthorFirstName $</$ CoMMA : FirstName $>$

$</$ COMMA : Person>

$</$ COMMA: CreatedBy $>$

$<$ CoMMA:Title>?DocTitle</CoMMA:Title $>$

$<$ COMMA : Edited $>$

$<$ COMMA: Company $>$

$<$ CoMMA : Designation $>\sim$ smith $</$ CoMMA : Designation $>$

$<$ COMMA: Situated $>$

$<$ COMMA:Location $>$

$<$ CoMMA : City $>$ Nice $</$ CoMMA : City $>$

$<$ CoMMA : Country $>$ France $</$ CoMMA : Country $>$

$<$ COMMA: Phone $>$ ?EditorPhone</COMMA: Phone $>$

$</$ COMMA: Location $>$

$</$ CoMMA: Situated $>$

$<$ CoMMA: HasForActivity $>$

$<$ COMMA:Edition / >

$</$ CoMMA:HasForActivity $>$

$</$ CoMMA : Company $>$

$</$ CoMMA : Edited $>$

$<$ COMMA : CreationDate $>\mathbf{2 0 0 0 < / C O M M A}$ : CreationDate $>$

$<$ COMMA : ReviewedBy $>$

$<$ COMMA : Person $>$

$<$ CoMMA : FamilyName $>$ hofstadter $</$ CoMMA : FamilyName $>$

$<$ CoMMA: FirstName $>$ douglas $</$ CoMMA: FirstName $>$

$</$ COMMA: Person $>$

$</$ CoMMA: ReviewedBy $>$

$<\mathrm{COMMA}:$ Concern $>$

$<$ COMMA:ComputerScienceTopic />

$</$ COMMA : Concern $>$

$</$ COMMA : Document $>$ 


\section{Solving / Decomposition}

Decomposition (Constraints)

- AM simplifies + decomposes $\rightarrow$ sub-queries to AA

$\checkmark$ Bottom-up constraints solving

- Top-down question solving

- URI as cut/joint points
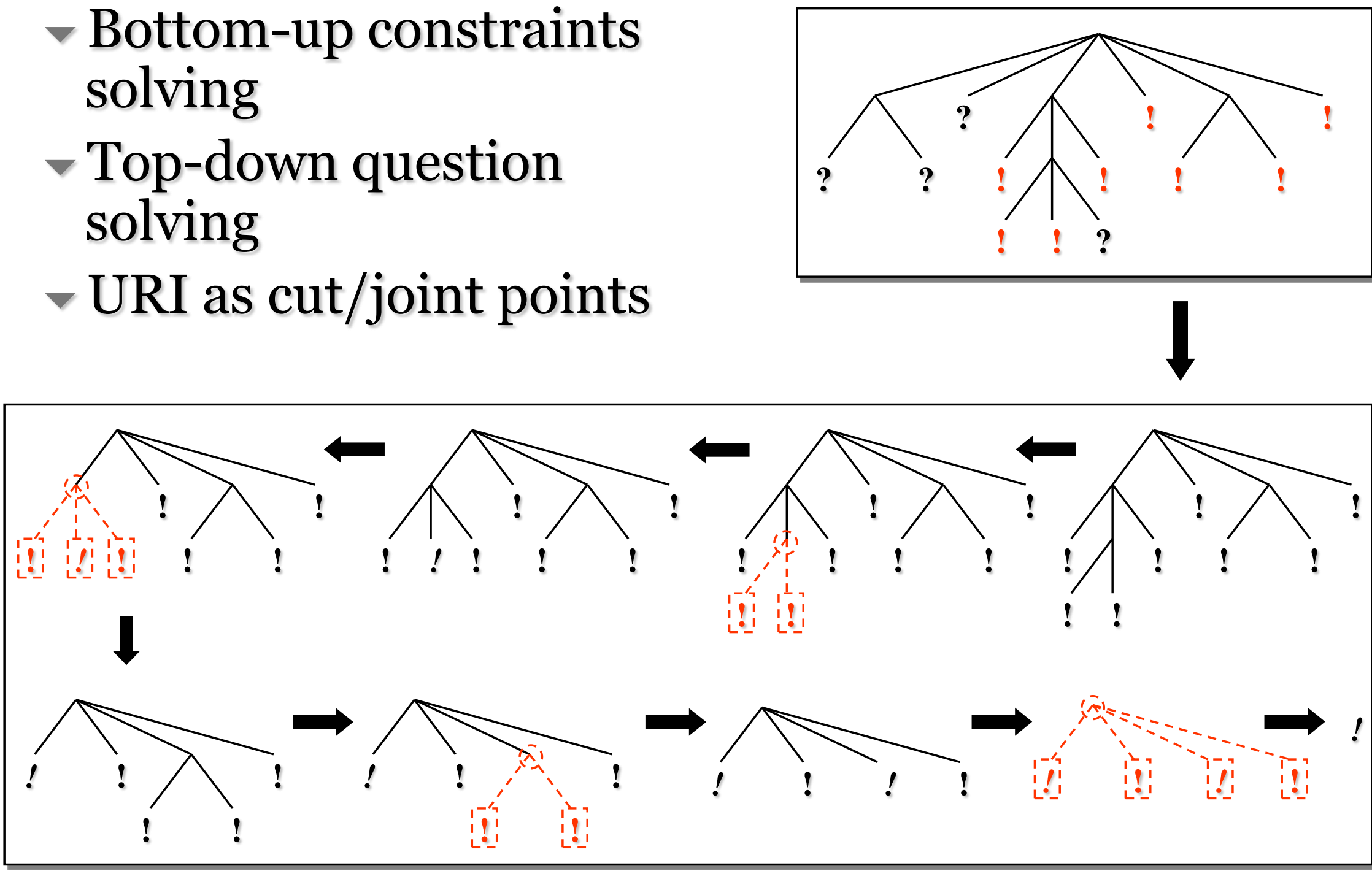
- AM simplifies + decomposes $\rightarrow$ sub-queries to AA

- Bottom-up constraints solving

- Top-down question solving

- URI as cut/joint points
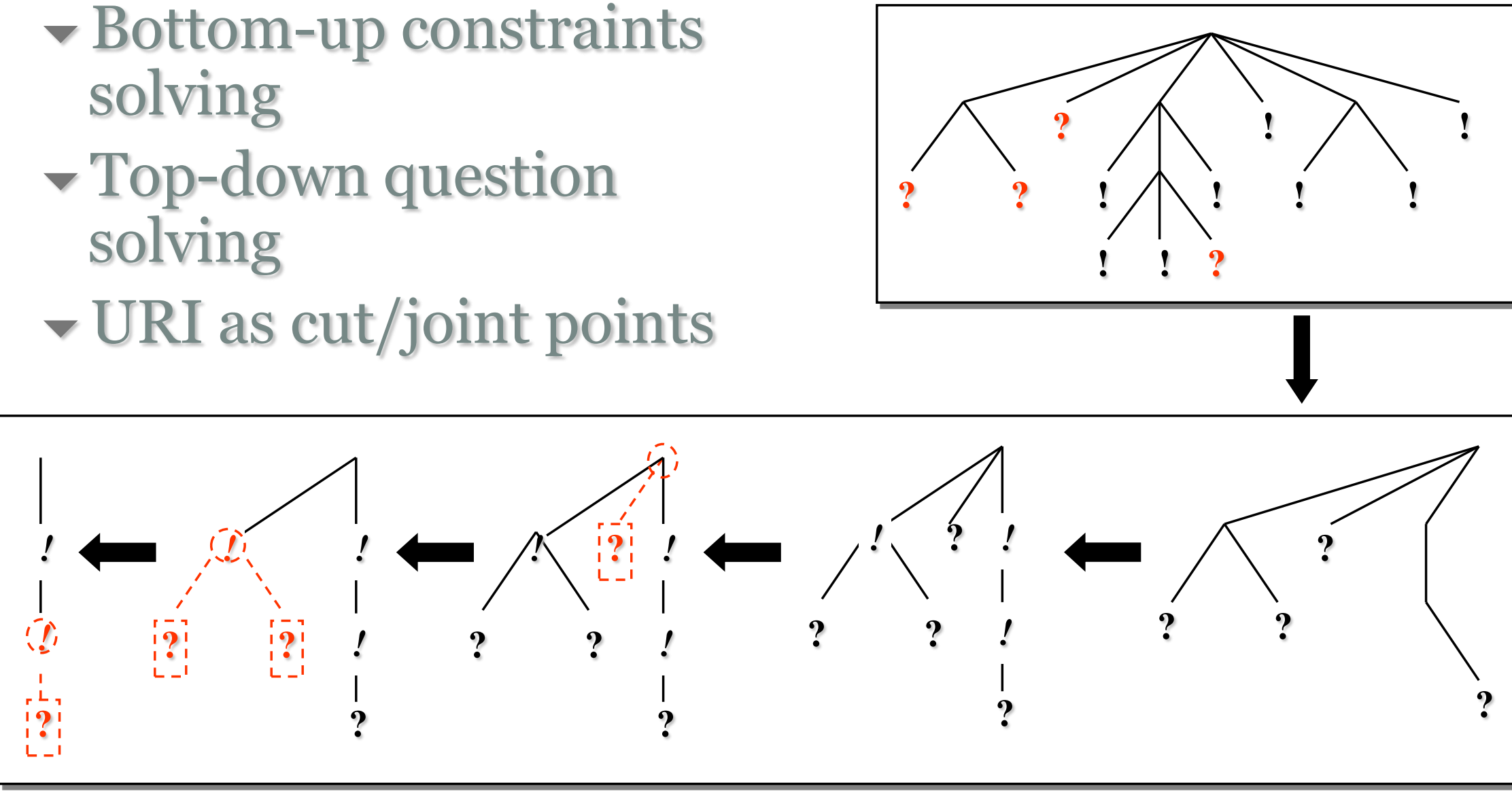

\section{Solving / Merging}

- AM merges partial results

- AM solves cross-references 
System implementation ( JNDED \& trial:

$\otimes$ Ergonomics problems and complexity

$\otimes$ Large scale real evaluation

(-) Working system i.e. proof of concept

(:) Usability and Usefulness recognized

(-) Developer appreciation of Agent-Onto coupling

(): Industrial interest in the $\mathrm{dvp}^{\mathrm{nt}}$ of the prototype

\section{Focused criticisms:}

- pseudo-semantic distance; literal analysis

- over specialisation; fine tuning, other criteria

- decomposition improvements (existential qualification, constraint focal point and heuristics)

Results $\in \mathrm{PhD}$ to be defended in October

Take home message: "looking for Post Doc." (;) 


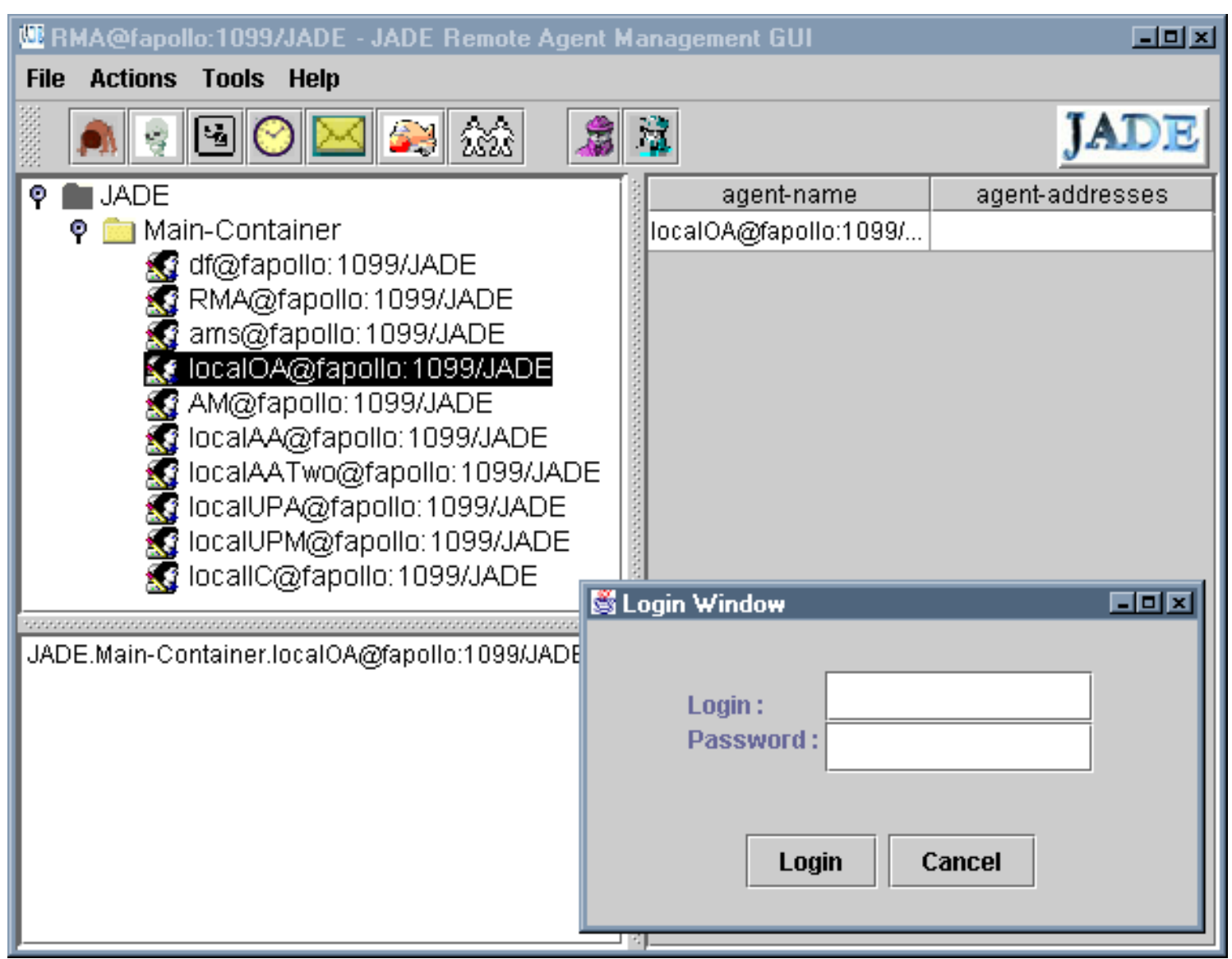




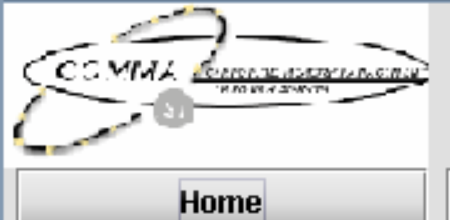

\section{CoIMVIA HIONE}

Ontology

Look for information

$$
Q \text { New query }
$$

$$
\text { Previous query... }
$$

Add information

I. New indexation

Previous indexation...

\section{Comments}

Amy problem ?

Contact us

About CohinA

$$
\text { Quit }
$$


CoMMA: Message passing in allocating an annotation

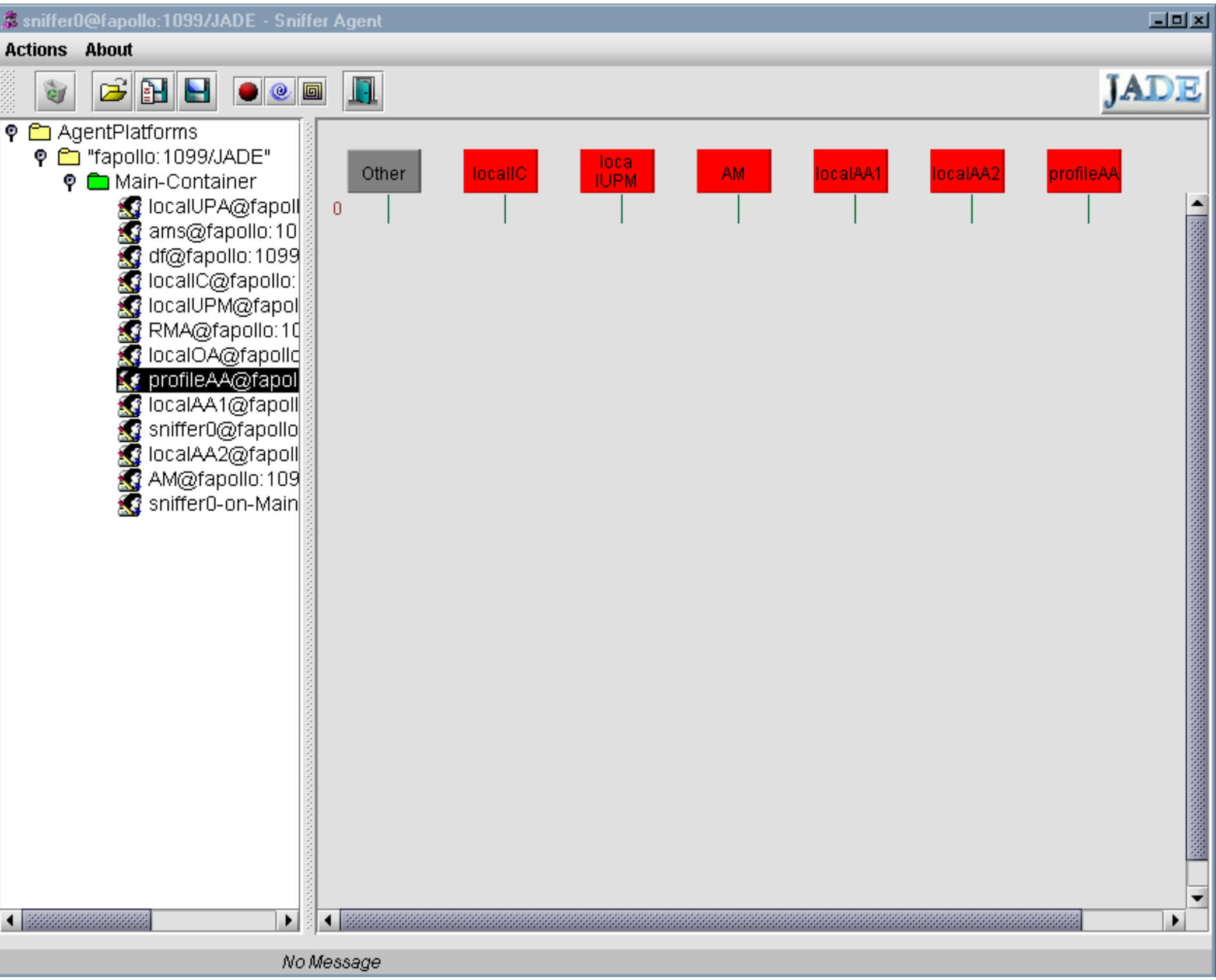



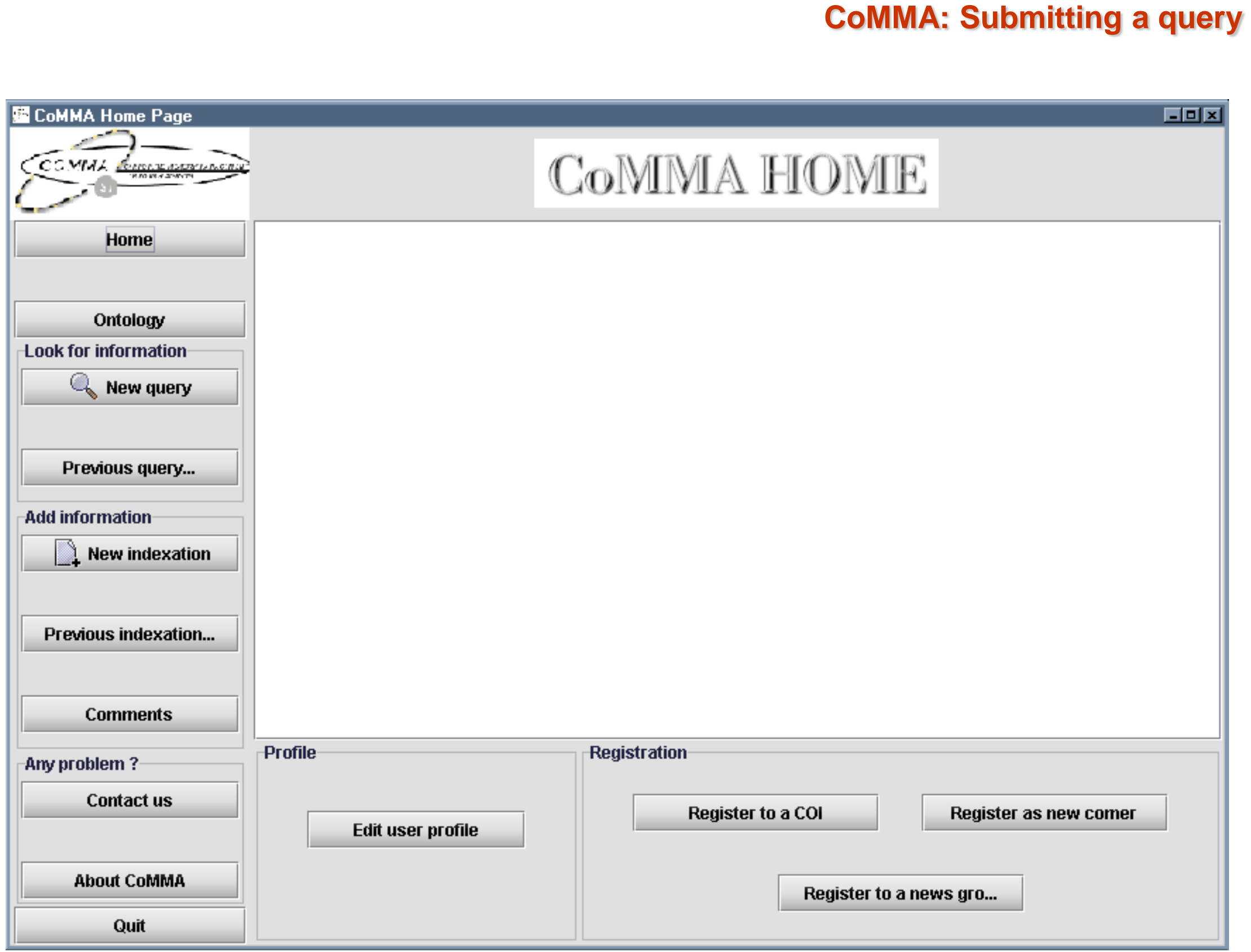


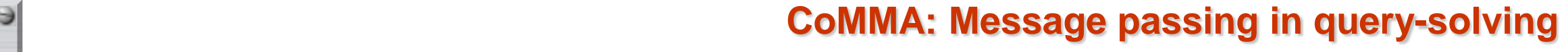

Actions About

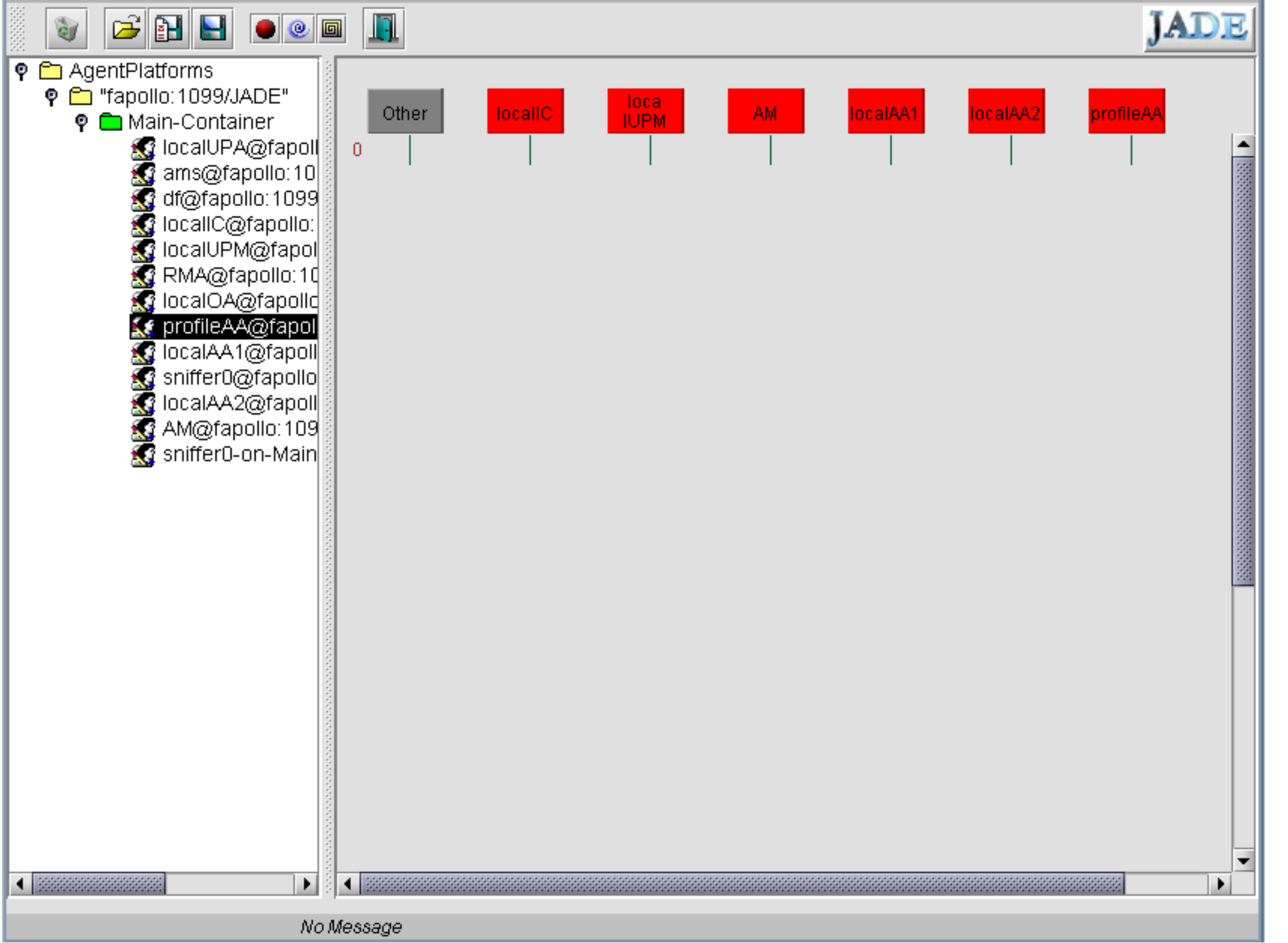




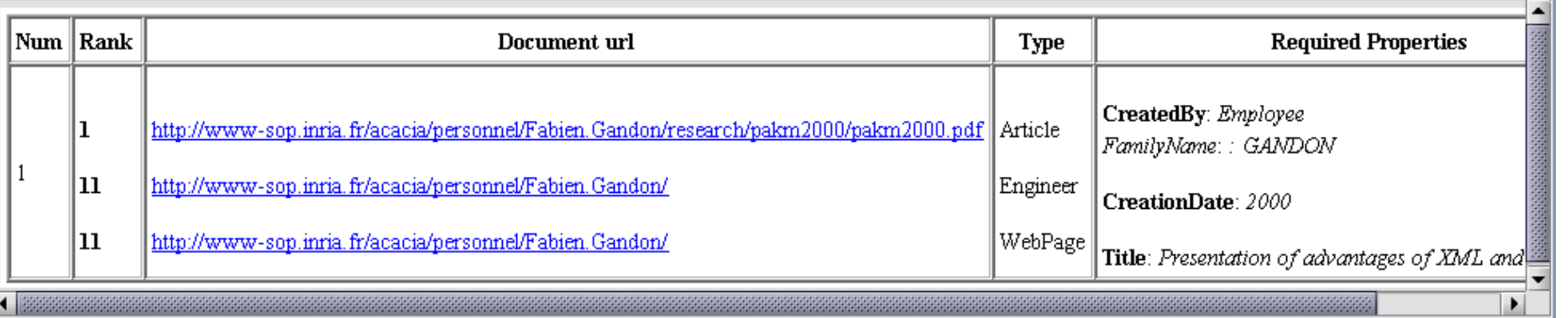




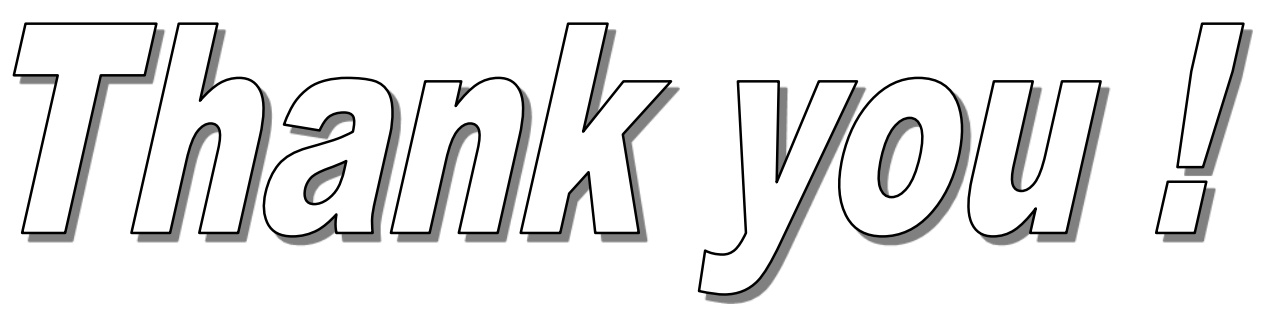

